\title{
基于 BIM 技术提高建筑工程管理效率的有效途径研究
}

\section{Research on Effective Ways to Improve Construction Engineering Management Efficiency Based on BIM Technology}

\author{
张禹 \\ Yu Zhang \\ 益科德(上海)有限公司 中国·辽宁 大连 200040 \\ Exyte (Shanghai) Co.,Ltd., Dalian, Liaoning, 200040, China
}

\begin{abstract}
摘 要: 随着城市化进展越来越快, 中国的建筑工程在各项建造工作之中担任着重要的角色。论文将对建筑工程之中如何使 用 BIM 技术来有效提高工程的管理质量进行相应的探讨和研究。论文首先简述 BIM 技术的主要内容, 然后说明中国建筑工 程的目前管理状况, 最后提出将 BIM 技术应用到建筑工程管理中的相应措施, 希望能够在一定程度上为中国建筑工作的发展 提供一些帮助。
\end{abstract}

\begin{abstract}
As urbanization progresses faster and faster, Chinese construction engineering plays an important role in various construction work. The paper will discuss and study how to use BIM technology in construction projects to effectively improve the management quality of projects. The paper first briefly describes the main content of BIM technology, then explains the current management status of China's construction engineering, and finally proposes corresponding measures to apply BIM technology to construction management, hoping to provide some help for the development of China's construction work to a certain extent.
\end{abstract}

关键词: BIM 技术; 建筑工程; 管理效率

Keywords : BIM technology; construction engineering; management efficiency

DOI : $10.36012 /$ etr.v2i7.2176

\section{1 引言}

在中国的建筑行业中,越来越多的科学技术被应用到了 建筑工程的施工或管理中,在一定程度上使整个建筑工程的 实际质量和施工工作内容得到了相应的保证。但是, 目前整 个建筑行业中已经存在的科学技术较为多样, 大多数技术还 没有得到合理应用, 这就导致这类科学技术不能发挥实际有 效的作用。对 BIM 技术的使用进行探讨, 能够对中国建筑工 程的管理工作有一定的提升和帮助。

\section{2 简述 BIM 技术的主要内容和优势}

所谓 BIM 技术, 就是通过对相应的建筑工程, 建立立体 的模拟信息模型来实现对建筑工程中设备、人力、资源、物力 等各个方面的有效管理。这样的模拟技术提高了整个工程的
管理效率, 并且能够实现较高合理度的管理配置, 能够对整 个工程设置可视化甚至透明化的管理规章制度, 对中国建筑 工程管理方式有一定的突破, 极大地提高了中国建筑工程管 理的效率。同时, BIM 技术的使用还具有以下优点:

$$
\text { 第一, BIM 技术可以极大地提高在建筑工程中对大量信 }
$$
息管理和处理的实际效率。由于整个建筑工程的实际规模较 大, 所以需要管理的相关数据和资源设备都是十分复杂的, 如果使用人力进行相应的管理工作和记录工作, 那么则无法 实现高效的管理效率。而 BIM 技术可以通过较为科学的技 术方法来对整个工程中所出现的相关信息进行收录和记载, 可以进一步提升管理工作的管理效率。

第二, 在传统的建筑工程项目的建设之前, 相关工作人 员都需要设计相应的施工图纸才能够进行接下来的施工工

【作者简介】张禹 (1984 ), 男, 辽宁大连人, 工程师, 从事工程管理研究。 
作, 传统的图纸不具备强烈的可观性, 而如果使用 BIM 技术 在建筑工程开展进行图纸信息的模拟,则可以实现将图纸可 视化、立体化的目标, 能够让参与到整个项目规划观看的工 作人员都可以观察到三维模拟所展示的施工图纸的实际效 果, 这样的展示方式能够让施工过程变得更加清晰明亮 [1]。

\section{3 目前中国建筑工程管理工作的大致状况}

第一, 无法实现高质量的建筑工程质量把控工作。由于 在施工过程中, 施工工人的施工水准没有相应的要求, 并且 在施工过程中有一部分的施工人员会随意使用施工现场的 建筑材料, 导致施工材料的数量不足以满足工程的建造的情 况发生, 这些都对工程的完成期限有很大的拖延, 并且影响 整个工程的质量。

第二, 在进行工程的施工时, 如果没有严格控制工程的 施工时间, 那么则会导致整个建筑工程的施工期限过长的情 况发生, 建筑商和开发商的相关利益将会受到损害, 那么整 个建筑工程所能够带来的实际收益也会受到许多外部因素 的影响。

第三,工程的成本也无法得到有效控制。管理制度和监督 力度都不符合相关建筑工程的实际要求, 所以大多数工程进 行建造的实际成本将会远远高于所预估的工程造价, 甚至在 许多工程施工的后期会出现成本不足以负担材料的消费、工人 的工资等情况,严重影响时将会拖延整个工程的实际进度和 质量。在生活里, 不难发现许多建筑物由于资金的缺失, 在后 期直接被放弃, 造成整个建筑工程烂尾的情况发生。所以, 目 前中国建筑工程的实际管理状况依然存在着许多问题 ${ }^{[2]}$ 。

\section{4 如何实现 BIM 技术在建筑工程管理中的 有效运用}

\section{1 在工程的计算管理中使用 BIM 技术}

实际建筑施工过程中, 大量的建筑工程数据都需要进行 计算, 利用 BIM 技术可以有效地进行工程计算管理工作。在 传统的建筑工程管理中, 相关人员基本都是采取普通的计算 器对工程存在的数据进行记录和计算, 这就导致工程的实际 数据状况无法得到有效的保证。而 BIM 技术可以使用强大 的计算能力, 确保整个建筑工程所涉及的数据都能够得到准 确的计算。在一定程度上降低了人力资源的浪费, 同时简化 了在施工过程中计算工作的相应难度, 也能够确保所计算出
的结果和内容符合相关标准和要求, 保证整个工程的数据计 算工作有很强的准确性 ${ }^{[3]}$ 。

\section{2 在工程的设计之中使用 BIM 技术}

在传统的设计管理工作上, 如果是单独的设计人员, 那 么基本不可能在极短的时间内完成一个大工程规模的相关 设计工作, 甚至在设计过程会出现很多与数据或者工程相关 信息的失误状况，这就导致整个工程的设计内容无法得到有 效性和正确性的保证。而将 BIM 技术运用于设计管理过程, 能够让设计人员建立可视化的模拟设计系统, 当设计人员需 要调整某一个地区的相应设计内容时, 直接可以调出相应 BIM 内容, 先对模型进行调整观察方案的可行性, 再实际的 放到建造工作中, 这样实现可视化的设计调整工作, 极大地 提高了整个设计工作的实际效率 ${ }^{[4]}$ 。

\section{3 在工程的资源分配中使用 BIM 技术}

在一个较大规模的工程项目中, 每一个资源的实际状况 都需要进行详细的记录, 但是这样的记录工作十分复杂, 并 且具有一定的难度, 需要在人力、物力、财力上投入较大的精 力。而 BIM 技术就可以在同一个系统内将所有的信息进行 统一的收集和归纳, 并且对信息进行相应的整理和处理,那 么就可以确保在建筑工程所出现的所有资源的管理工作可 以得到有效地提升。

\section{5 结语}

BIM 技术的使用可以在建筑工程管理工作的方方面面 得到体现,也提升了整个建筑工程的管理效率。而相关工作 人员需要详细了解 BIM 使用方向和范围, 在正确方法方式 的指导下，充分发挥 BIM 技术在项目工程管理过程中所具 有的作用, 推动中国项目工程的管理工作向前发展和进步。

\section{参考文献}

[1] 谢蹈.基于 BIM 技术提高建筑工程管理效率的有效途径研究 [J]. 商讯,2020(6):1 66+168.

[2] 曾峰. 论 BIM 技术在建筑工程管理中的应用[J].中国标准化, 2018(22):17-18.

[3] 王中琴.建筑工程管理应用 BIM 技术的效率提高方法分析[J]. 信息记录材料,2018(12):94-95.

[4] 马联华,黄敏. 基于 BIM 技术提高建筑工程管理效率的有效途 径探索[J].科技经济导刊,2019(9):49. 